Research article

\title{
Saline stress enhanced accumulation of leaf phenolics in honeysuckle (Lonicera japonica Thunb.) without induction of oxidative stress
}

\author{
Kun Yan ${ }^{\mathrm{a},}{ }^{*}$, Shijie Zhao ${ }^{\mathrm{b}}$, Lanxing Bian ${ }^{\mathrm{c}}$, Xiaobing Chen ${ }^{\mathrm{a}}$ \\ ${ }^{\text {a }}$ Key Laboratory of Coastal Environmental Processes and Ecological Remediation, Yantai Institute of Coastal Zone Research, Chinese Academy of Sciences, \\ Yantai, China \\ b State Key Laboratory of Crop Biology, Shandong Agricultural University, Tai'an, China \\ ${ }^{\mathrm{c}}$ College of Life Sciences, Yantai University, Yantai, China
}

\section{A R T I C L E I N F O}

\section{Article history:}

Received 7 November 2016

Received in revised form

28 December 2016

Accepted 21 January 2017

Available online 22 January 2017

\section{Keywords:}

Antioxidant enzyme

Ascorbate

Phenylalanine ammonia-lyase

Phenolics

Photosynthetic electron transport

\begin{abstract}
A B S T R A C T
Honeysuckle (Lonicera japonica Thunb.) is a traditional medicinal plant in Chinese, and chlorogenic acid and luteolosid are its specific bioactive phenolic compounds. This study was to investigate leaf antioxidant responses in honeysuckle to saline stress with emphasis on phenolics through hydroponic experiments and field trials. $\mathrm{NaCl}$ stress did not stimulate antioxidant system including superoxide dismutase, ascorbate peroxidase, catalase and ascorbate, and had no significant effect on lipid peroxidation in the leaves. Consistently, no inhibition on photochemical capacity of photosystems suggested that reactive oxygen species (ROS) was maintained at a normal level under $\mathrm{NaCl}$ stress. However, leaf phenolic synthesis was activated by $\mathrm{NaCl}$ stress, indicated by elevated genes transcription and activity of phenylalanine ammonia-lyase and increased phenolics concentration. Specifically, leaf chlorogenic acid concentration was increased by $67.43 \%$ and $48.86 \%$ after 15 days of 150 and $300 \mathrm{mM} \mathrm{NaCl}$ stress, and the increase of luteolosid concentration was $54.26 \%$ and $39.74 \%$. The accumulated phenolics hardly helped detoxify ROS in vivo in absence of oxidative stress, but the elevated phenolic synthesis might restrict ROS generation by consuming reduction equivalents. As with $\mathrm{NaCl}$ stress, soil salinity also increased concentrations of leaf phenolics including chlorogenic acid and luteolosid without exacerbated lipid peroxidation. In conclusion, leaf phenolics accumulation is a mechanism for the acclimation to saline stress probably by preventing oxidative stress in honeysuckle; leaf medicinal quality of honeysuckle can be improved by saline stress due to the accumulation of bioactive phenolic compounds.
\end{abstract}

๑ 2017 Elsevier Masson SAS. All rights reserved.

\section{Introduction}

Salt stress causes ion homeostasis disruption in plant cells and results in oxidative damage with excess generation of reactive oxygen species (ROS) (Gill and Tuteja, 2010; Munns and Tester, 2008; Zhu, 2016). In photosynthetic organisms, the major ROS generation sites are located at the reaction centers of photosystem I and II (PSI and PSII) in chloroplast (Asada, 2006). In particular, superoxide

Abbreviations: APX, ascorbate peroxidase; AsA, ascorbate; CAT, catalase; ETR, electron transport rate; Fv/Fm, the maximal photochemical capacity of PSII; $\mathrm{H}_{2} \mathrm{O}_{2}$, hydrogen peroxide; MDA, malondialdehyde; NADPH, nicotinamide adenine dinucleotide phosphate; $\mathrm{O}_{2}{ }^{*}$, superoxide anion; PAL, phenylalanine ammonia-lyase; Pn, Photosynthetic rate; PSI, photosystem I; PSII, photosystem II; ROS, reactive oxygen species; SOD, superoxide dismutase; $\triangle M R / M_{0}$, the maximal photochemical capacity of PSI.

* Corresponding author.

E-mail addresses: kyan@yic.ac.cn, yankunacademic@163.com (K. Yan). anion $\left(\mathrm{O}_{2}{ }^{-}\right)$tends to be generated through linear photosynthetic electron transport due to nicotinamide adenine dinucleotide phosphate (NADPH) accumulation, when $\mathrm{CO}_{2}$ assimilation is inhibited under abiotic stresses (Takahashi and Murata, 2008). Subsequently, $\mathrm{O}_{2}{ }^{-}$is transformed to hydrogen peroxide $\left(\mathrm{H}_{2} \mathrm{O}_{2}\right)$ through dismutation reaction, and finally, the most dangerous ROS, hydroxyl radical, is generated through Fenton reaction (Gill and Tuteja, 2010).

Plants have evolved a suite of antioxidant system to protect against oxidative damage by scavenging ROS. The antioxidant system consists of: (1) metabolites, the most abundant being ascorbate (AsA); (2) ROS scavenging enzymes, such as superoxide dismutase (SOD), catalase (CAT) and ascorbate peroxidase (APX) (Gill and Tuteja, 2010). SOD catalyzes the dismutation of $\mathrm{O}_{2}^{-} \cdot$ to $\mathrm{H}_{2} \mathrm{O}_{2}$, which is subsequently scavenged by APX and CAT, and AsA can directly scavenge ROS or act as reducing substrate for APX to detoxify $\mathrm{H}_{2} \mathrm{O}_{2}$ (Gill and Tuteja, 2010). Thus, they work together to 
limit the generation of hydroxyl radical by controlling the levels of $\mathrm{O}_{2}^{-} \cdot$ and $\mathrm{H}_{2} \mathrm{O}_{2}$. Under saline stress, plant antioxidant system is often stimulated to defense oxidative injury, and antioxidant capacity has been used as a criterion for the comparison of salt tolerance among crop cultivars (Ashrafi et al., 2015; Kiani-Pouya, 2015; Zhang et al., 2014). Phenolic compounds which are secondary metabolites have high ROS scavenging capacity, and accumulation of phenolics is commonly considered as a positive self-protection way to strengthen ROS scavenging ability in plants upon salt-induced oxidative stress (Abrol et al., 2012; Colla et al., 2013; Falleh et al., 2012; Ksouri et al., 2007; Shao et al., 2015; Zhao et al., 2015). Nevertheless, antioxidant activity of phenolic compounds is mainly proved by in vitro free radical scavenging experiments, and their roles in mitigating oxidative stress in vivo are still open to debate (Hernandez et al., 2009; Hernandez and Van Breusegem, 2010). Phenolic compounds are mainly conserved in vacuoles, and can scavenge ROS diffused from chloroplasts under severe oxidative stress (Ferreres et al., 2011; Niggeweg et al., 2004). Thus, as a traditional viewpoint, phenolic compounds are defined as secondary antioxidants, and play an assisting role in detoxifying ROS, when the primary antioxidant system is depressed under severe stress (Fini et al., 2011). However, this traditional viewpoint has been challenged recently, because moderate saline stress is more liable to induce accumulation of phenolics in plants without severe oxidative stress and depression of antioxidant system (Abrol et al., 2012; Karray-Bouraoui et al., 2010; Ksouri et al., 2007; Shao et al., 2015; Zhao et al., 2015). Therefore, the relation between antioxidant system and phenolic metabolites in resisting oxidative stress is still not clear.

Honeysuckle (Lonicera japonica Thunb.) is a twining semievergreen vine native to East Asia, and chlorogenic acid and luteolosid are its specific bioactive phenolic compounds. In Chinese, honeysuckle is a traditional medicinal plant, and has been widely used in healthcare, food and cosmetics industries. At present, studies attach importance to salt-induced changes in concentrations of bioactive phenolic compounds in medicinal plants (Colla et al., 2013; Karray-Bouraoui et al., 2010; Shao et al., 2015; Zhao et al., 2015). However, antioxidant mechanisms of phenolics in vivo are often overlooked, and the relation between accumulation of phenolics and antioxidant system induction under saline stress is less concerned. In addition, salt-induced accumulation of phenolics in plants is majorly demonstrated by hydroponic experiments and unusually evidenced by field trials. Especially, Bautista et al. (2016) reported that plant phenolics concentration was weakly correlated with soil salinization in natural habitat. Thus, salt-induced accumulation of phenolics in plants lacks ecological proof and practical value.

In recent years, we identified a salt tolerant honeysuckle cultivar and demonstrated its phytoremediation effect on saline soil (Yan et al., 2015, 2016). In this study, we aimed to explore leaf antioxidant responses in honeysuckle under saline stress with emphasis on phenolics through hydroponic experiments and field trials. We hypothesized that the accumulation of leaf phenolics could be improved in honeysuckle to protect against oxidative damage under saline stress by assisting antioxidant system to scavenge ROS.

\section{Materials and methods}

\subsection{Hydroponic experiments}

Bare-rooted honeysuckle plants were planted in plastic pots filled with quartz sand. The plants were watered with Hoagland solution ( $\mathrm{pH}$ 5.7) and placed in a climatic chamber (Qiushi, China). The photon flux density, day/night temperature and humidity were controlled at $200 \mu \mathrm{mol} \mathrm{m}^{-2} \mathrm{~s}^{-1}$ (12 h per day from 07:00 to 19:00),
$25 / 18{ }^{\circ} \mathrm{C}$ and $65 \%$ in the chamber. After 60 days, healthy and uniform plants were selected for salt treatments. $\mathrm{NaCl}$ was added to nutrient solution incrementally by daily $50 \mathrm{mM}$ steps to final concentrations of 150 and $300 \mathrm{mM}$. Nutrient solution without adding $\mathrm{NaCl}$ was used for the cultivation of control plants. After 0,7 and 15 days of salt stress, the newly expanded leaves were sampled from four replicate plants in each treatment for the measurements of physiological parameters.

\subsection{Field trials}

The experiment site was established in Dongying Halophyte Arboretum, Dongying Academy of Agricultural Sciences, Shandong province, China $\left(37^{\circ} 24^{\prime} \mathrm{N}, 118^{\circ} 39^{\prime} \mathrm{E}\right.$ and $8.8 \mathrm{~m}$ above sea level). This area has a warm temperate continental monsoon climate. The annual average temperature and precipitation at this site are $12.8{ }^{\circ} \mathrm{C}$ and $555.9 \mathrm{~mm}$, respectively.

Bare-rooted honeysuckle plants were planted in non-saline area in the arboretum in November, 2013. Four replicate plots $(3 \mathrm{~m} \times 4 \mathrm{~m}$ ) were constructed in non-saline and saline areas. The initial soil nutrients, salinity and $\mathrm{pH}$ were reported in our recent study (Yan et al., 2016). The average electronic conductance and sodium adsorption ratio were $486 \mu \mathrm{sm}^{-1}$ and 9.51, respectively, in non-saline plots and $910 \mu \mathrm{s} \mathrm{cm}^{-1}$ and 16.43 , respectively, in saline plots. To avoid border effects, an isolation belt of $0.5 \mathrm{~m}$ was left around the plots. The plots were ploughed, and compound fertilization was applied at $750 \mathrm{~kg} \mathrm{ha}^{-1}$. In April, 2014, 45 plants were transplanted to each plot; plant and row spacing was $0.5 \mathrm{~m}$ and $0.75 \mathrm{~m}$. The newly expanded leaves were sampled from three randomly selected plants in each plot in growth seasons of 2014 and 2015.

\subsection{Measurements of photosynthetic parameters}

Photosynthetic rate (Pn) and electron transport rate (ETR) were detected by using an open photosynthetic system (LI-6400XTR, LiCor, Lincoln, NE, USA) equipped with a fluorescence leaf chamber (6400-40 LCF, Li-Cor). Temperature, $\mathrm{CO}_{2}$ concentration and actinic light intensity were, respectively, set at $25{ }^{\circ} \mathrm{C}, 400 \mu \mathrm{mol} \mathrm{mol}^{-1}$ and $1000 \mu \mathrm{mol} \mathrm{m}{ }^{-2} \mathrm{~s}^{-1}$ in the leaf cuvette. Pn was recorded, when it reached a steady level. Steady-state fluorescence yield was also recorded, and then a saturating actinic light pulse of $8000 \mu \mathrm{mol} \mathrm{m}{ }^{-2} \mathrm{~s}^{-1}$ for $0.7 \mathrm{~s}$ was used to produce maximum fluorescence yield by temporarily inhibiting PSII photochemistry for measuring actual photochemical efficiency of PSII ( $\Phi P S I I)$. ETR were calculated as $\Phi$ PSII $\times$ PPFD $\times 0.84 \times 0.5$ (Maxwell and Johnson, 2000).

A multifunctional plant efficiency analyzer (MPEA, Hansatech, UK) was used to measure the maximal photochemical capacity of PSI $\left(\Delta \mathrm{MR} / \mathrm{MR}_{0}\right)$ and PSII (Fv/Fm) (Yan et al., 2013a). The leaves were kept in the dark for 30 min before the measurements. Thereafter, the leaves were orderly illuminated with $1 \mathrm{~s}$ red light $(627 \mathrm{~nm}$, $5000 \mu \mathrm{mol}$ photons $\mathrm{m}^{-2} \mathrm{~s}^{-1}$ ), $10 \mathrm{~s}$ far red light $(735 \mathrm{~nm}, 200 \mu \mathrm{mol}$ photons $\left.\mathrm{m}^{-2} \mathrm{~s}^{-1}\right)$ and $2 \mathrm{~s}$ red light $(627 \mathrm{~nm}, 5000 \mu \mathrm{mol}$ photons $\mathrm{m}^{-2} \mathrm{~s}^{-1}$ ). Chlorophyll fluorescence and modulated $820 \mathrm{~nm}$ reflection were simultaneously recorded during the illumination. Monitoring modulated reflection change near $820 \mathrm{~nm}$ is a very convenient way to follow redox state of PSI. The relative value of maximal difference of $820 \mathrm{~nm}$ reflection during the last $2 \mathrm{~s}$ red illumination was used to indicate $\Delta \mathrm{MR} / \mathrm{MR}_{0}$ (Schansker et al., 2003). Chlorophyll fluorescence transients were quantified to calculate Fv/Fm (Kalaji et al., 2014). 
2.4. Measurements of antioxidant enzymes activities, AsA content and redox state and malondialdehyde (MDA) content

The methods of extraction and measurement have been illuminated in detail in our previous study (Yan et al., 2010). SOD activity ( $\mathrm{U} \mathrm{g}^{-1} \mathrm{FW}$ ) was determined by the inhibition on nitro blue tetrazolium reduction. APX ( $\mu$ mol AsA $\min ^{-1} \mathrm{~g}^{-1} \mathrm{FW}$ ) and CAT ( $\mathrm{mmol} \mathrm{H} \mathrm{O}_{2} \mathrm{~min}^{-1} \mathrm{~g}^{-1} \mathrm{FW}$ ) activities were, respectively, estimated by AsA oxidation rate and $\mathrm{H}_{2} \mathrm{O}_{2}$ decomposing rate. AsA content ( $\mathrm{mg} \mathrm{g}^{-1} \mathrm{FW}$ ) was detected by using a high performance liquid chromatograph (Thermo, USA) with a hypersil C18 column $(5.0 \mu \mathrm{m}$ particles size, $4.6 \times 150 \mathrm{~mm}$ ). Dithiothreitol was used to completely reduce the oxidized AsA for detecting total AsA content and calculating AsA redox state. MDA content was reckoned by the reaction with thiobarbituric acid.

\subsection{Measurements of phenolic concentration and DPPH scavenging capacity}

Leaves were dried at $40{ }^{\circ} \mathrm{C}$ to constant weight, and ground to pass through $0.25 \mathrm{~mm}$ sieve for measuring phenolics concentration. Dry leaf powder $(0.1 \mathrm{~g})$ was homogenized in $10 \mathrm{ml}$ 60\% (V/V) methanol, and ultrasonic extraction was carried out at $40{ }^{\circ} \mathrm{C}$ for $40 \mathrm{~min}$. The mixture was centrifuged for $10 \mathrm{~min}$ at $10000 \times \mathrm{g}$. The supernatant $(0.1 \mathrm{ml})$ was mixed with $0.15 \mathrm{ml}$ Folin-Ciocalteau reagent, $0.15 \mathrm{ml}$ of $10 \%(\mathrm{~W} / \mathrm{V}) \mathrm{Na}_{2} \mathrm{CO}_{3}$ and $4.6 \mathrm{ml}$ of distilled water. After 80 min incubation at room temperature in the dark, the absorbance at $760 \mathrm{~nm}$ was measured. Total phenolics content was determined by using a standard curve plotted with known concentrations of gallic acid (Colla et al., 2013). Flavonoid content was measured by using differential spectrophotometry method with $\mathrm{ZrOCl}_{2} \cdot 8 \mathrm{H}_{2} \mathrm{O}$ for avoiding the disturbance of chlorogenic acid (Zhou et al., 2007). The supernatant $(0.2 \mathrm{ml})$ was mixed with $1.5 \mathrm{ml}$ $\mathrm{ZrOCl}_{2} \cdot 8 \mathrm{H}_{2} \mathrm{O}(0.2 \%)$ and $3.3 \mathrm{ml}$ methanol, and another $0.2 \mathrm{ml}$ supernatant was mixed with $4.8 \mathrm{ml}$ methanol. Then, the mixtures were incubated at room temperature in the dark for $1 \mathrm{~h}$, and the differences of absorbance at $410 \mathrm{~nm}$ were determined. Flavonoid content was calculated by using a standard curve plotted with known concentrations of lutin.

Chlorogenic acid and luteoloside concentrations were assayed according to Zhang et al. (2007) with some modification. The assay was performed in a high performance liquid chromatography system (Thermo, USA) with a hypersil C18 column $(5.0 \mu \mathrm{m}$ particles size, $4.6 \mathrm{~mm} \times 150 \mathrm{~mm}$ ). The supernatant was filtered through a $0.45 \mu \mathrm{m}$ membrane filter before injecting into the column; the injection volume was $10 \mu \mathrm{l}$. The mobile phase consisted of $0.3 \%(\mathrm{v} / \mathrm{v})$ formic acid aqueous solution (A) and acetonitrile (B), and a gradient elution program was applied as follows: at $0 \mathrm{~min}$, the volume ratio between $A$ and $B$ was $92 / 8$, and the ratio was changed to $83 / 17$ from 0 to $12 \mathrm{~min}$, subsequently to $80 / 20$ from 16 to $21 \mathrm{~min}$, and finally returned to $92 / 8$ at $22 \mathrm{~min}$ through a linear gradient. The flow rate was $1.0 \mathrm{ml} \mathrm{min}-1$, and the column temperature was $38{ }^{\circ} \mathrm{C}$. Chlorogenic acid and luteoloside in samples were identified by comparing their retention times in UV spectra with those of the standards (Fig. 1). Chlorogenic acid and luteoloside were, respectively, detected at $330 \mathrm{~nm}$ and $360 \mathrm{~nm}$, and their concentrations were determined by using a standard curve plotted with known concentrations of the standards.

The diluted supernatant $(0.3 \mathrm{ml})$ and pure methanol $(0.3 \mathrm{ml})$ were, respectively, mixed with $60 \mu \mathrm{M}$ DPPH methanol solution (3 $\mathrm{ml})$. The mixture was shacked vigorously and incubated for 30 min in the dark at room temperature, and then the absorbance at $517 \mathrm{~nm}$ was assayed (Khan et al., 2013). DPPH scavenging rate (\%) was calculated as $\left(A_{\text {blank }}-A_{\text {sample }}\right) / A_{\text {blank }} \times 100$, where $A_{\text {sample }}$ and

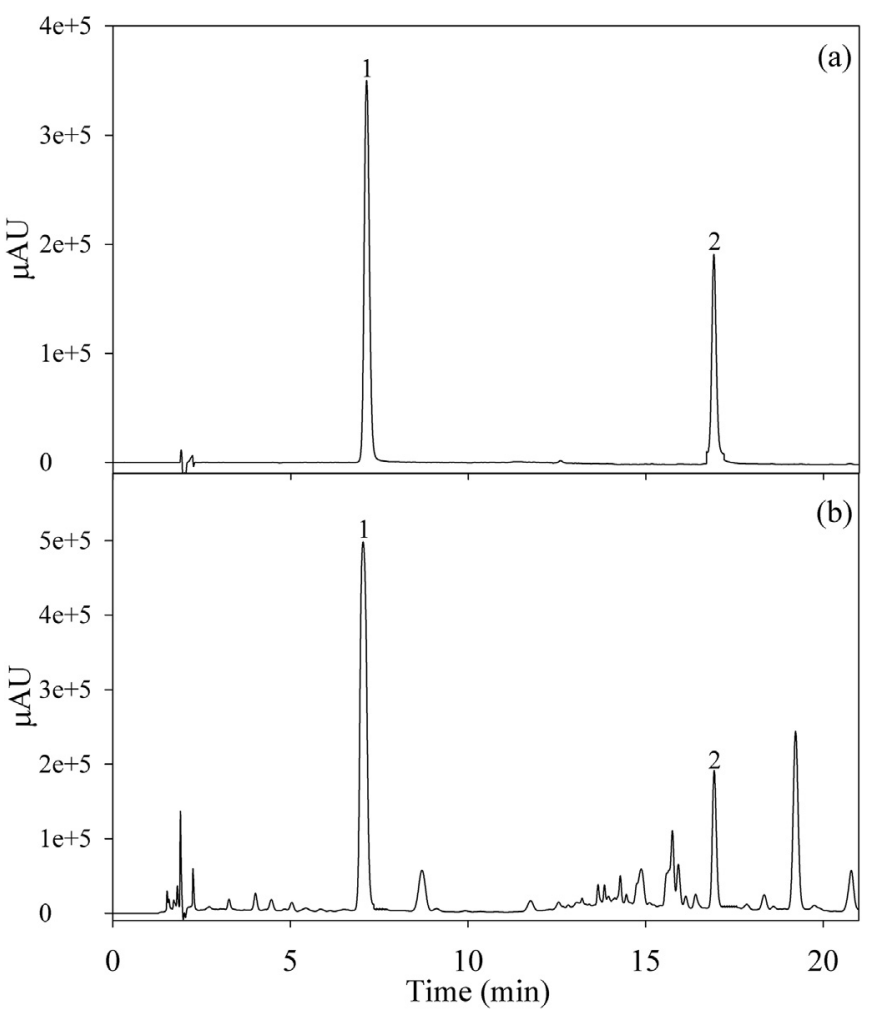

Fig. 1. High performance liquid chromatogram of chlorogenic acid (peak 1) and luteolosid (peak 2) of standards (a) and a leaf sample (b).

Ablank were the absorbance of mixtures in the presence and absence of plant extract, respectively.

\subsection{Measurement of phenylalanine ammonia-lyase (PAL) activity}

Plant tissues $(0.2 \mathrm{~g})$ were ground under liquid nitrogen, and homogenized in $5 \mathrm{ml}$ of $50 \mathrm{mM}$ borate buffer ( $\mathrm{pH} 8.8$ ) containing $20 \mathrm{mM} \beta$-mercaptoethanol, 5\% (v/w) PVP, 1 mM EDTANa 2 and 0.1\% $(\mathrm{v} / \mathrm{w})$ Triton $\mathrm{X}-100$. The homogenate was centrifuged at $10000 \times \mathrm{g}$ and $4{ }^{\circ} \mathrm{C}$ for $20 \mathrm{~min}$, and the supernatant was collected. For determining PAL activity, $0.2 \mathrm{ml}$ supernatant was mixed with $2.8 \mathrm{ml}$ $0.02 \mathrm{M} \mathrm{L}$-phenylalanine (dissolved in $50 \mathrm{mM}$ borate buffer at $\mathrm{pH}$ 8.8 ), and incubated for $1 \mathrm{~h}$ at $30^{\circ} \mathrm{C}$. The increase in $\mathrm{OD}_{290 \mathrm{~nm}}$ due to the formation of cinnamate acid was measured, and PAL activity was expressed as the change in $\mathrm{OD}_{290 \mathrm{~nm} \mathrm{~h}} \mathrm{~h}^{-1} \mathrm{mg}^{-1}$ protein (Lister et al., 1996). Protein was estimated by coomassie brilliant blue staining with bovine serum albumin as a standard.

\subsection{Real time quantitative PCR analysis}

For real time expression analysis, the PCR mix contained $1 \mathrm{ml}$ of diluted cDNA (10 ng), $10 \mathrm{ml}$ of SYBR Green PCR Master Mix (Applied Biosystems, USA) and $200 \mathrm{nM}$ of specific primers in a final volume of $20 \mu \mathrm{l}$. Actin (ACT) (GenBank Accession No. GQ241342) was used as an internal reference gene to calculate relative transcript levels. The primers for ACT, PAL1, PAL2 and PAL3 are listed in Table 1. All PCRs were performed using a Fast Real Time PCR System (Applied Biosystems, USA) under the following conditions: 2 min at $95^{\circ} \mathrm{C}, 40$ cycles of $15 \mathrm{~s}$ at $95^{\circ} \mathrm{C}$, and $60 \mathrm{~s}$ at $60^{\circ} \mathrm{C}$ in optical 96 -well reaction plates. The specificity of amplicons was verified by melting curve analysis. Three technical replicates were analyzed for each gene. 
Table 1

Primers for real time quantitative PCR. PAL indicates the gene of phenylalanine ammonia-lyase. PAL gene family consists of PAL1, PAL2 and PAL3. Actin (ACT) is an internal reference gene.

\begin{tabular}{lll}
\hline Gene & Sense primer & Antisense primer \\
\hline ACT & CCAGTATTGTAGGTAGACCAAGAC & TCAATGGGGTATTTCAAGGTAAGG \\
PAL1 & GCCAATCCAGTCACTAACC & CGTAAATTCTCCTCCAAATGC \\
PAL2 & GCTCGCCCTTGTTAATGG & GTGGTGCTTCAACTTATGC \\
PAL3 & TGAACGCTGGAATCTTTGG & GGTGATGTTGTGGTTGAGG \\
\hline
\end{tabular}

\subsection{Statistical analysis}

One-way ANOVAs were carried out by using SPSS 16.0 (SPSS Inc., Chicago, IL, USA) for all sets of data. The values presented are the means of samples collected from four replicate plants in hydroponic experiments and four replicate plots in field trials. The comparisons of means were determined using a least significant difference test, and the differences were considered significant at $P<0.05$.

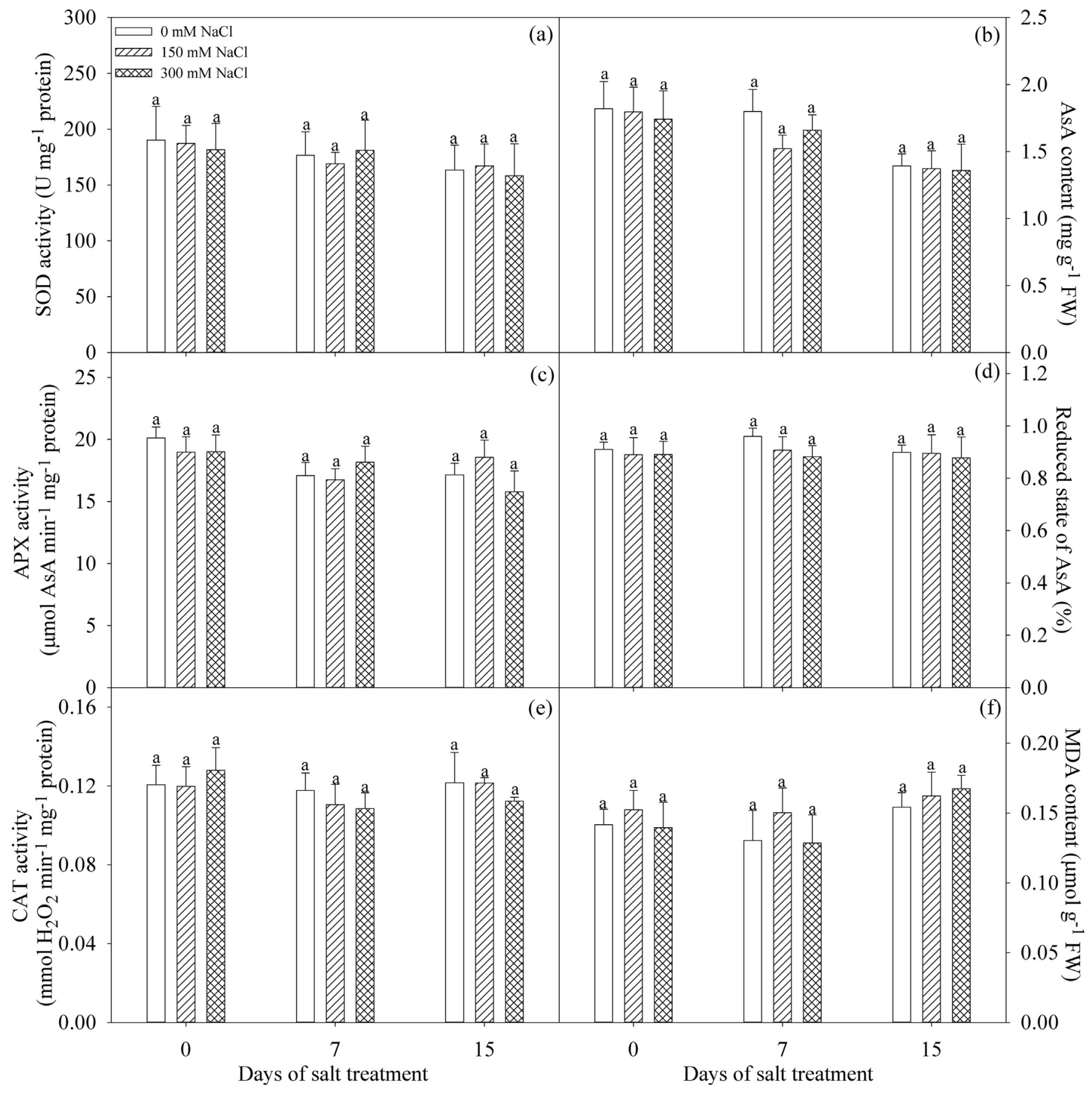

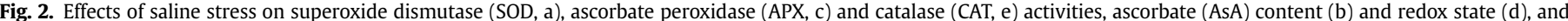

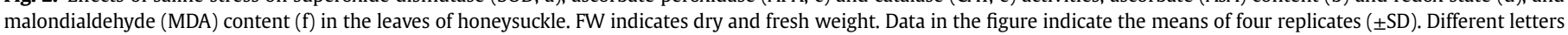
on error bars indicate salt-induced significant differences at $P<0.05$. 


\section{Results}

\subsection{Antioxidant system and MDA content under $\mathrm{NaCl}$ stress in hydroponic experiments}

After 7 and 15 days of $\mathrm{NaCl}$ stress, SOD, APX and CAT activities in the leaves were not significantly affected, as were MDA content and AsA content and redox state (Fig. 2). Thus, antioxidant system was not stimulated by $\mathrm{NaCl}$ stress.

\subsection{Photosynthetic rate and chlorophyll fluorescence parameters under $\mathrm{NaCl}$ stress in hydroponic experiments}

Pn was significantly decreased by $14.56 \%$ and $32.45 \%$ after 7 days of 150 and $300 \mathrm{mM} \mathrm{NaCl}$ stress, and the decrease was up to $29.93 \%$ and $60.22 \%$ at day 15 (Fig. 3a). ETR was significantly decreased by $17.28 \%$ and $27.53 \%$ after 7 days of 150 and $300 \mathrm{mM} \mathrm{NaCl}$ stress, and the decrease reached $21.30 \%$ and $50.02 \%$ at day 15 (Fig. 3b). Fv/Fm and $\Delta M R / M_{0}$ were not markedly affected after 7 and 15 days of $\mathrm{NaCl}$ stress (Fig. 3c and d).

\subsection{Phenolics concentration, PAL activity and DPPH scavenging rate} under $\mathrm{NaCl}$ stress in hydroponic experiments

Significant increases in concentrations of leaf total phenolics and flavonoid were noted after 7 days of $\mathrm{NaCl}$ stress, and the increases became greater when $\mathrm{NaCl}$ stress was prolonged to 15 days (Fig. 4a and b). Consistently, leaf chlorogenic acid and luteoloside concentrations were also significantly increased after 7 days of $\mathrm{NaCl}$ stress, and at day 15 , the increase of chlorogenic acid concentration was up to $67.43 \%$ and $48.86 \%$, respectively, under 150 and $300 \mathrm{mM}$ $\mathrm{NaCl}$ stress, while the increase of luteoloside concentration was $54.26 \%$ and $39.74 \%$, respectively (Fig. $4 \mathrm{c}$ and d). Remarkable increases in PAL activity and DPPH scavenging rate were observed after 7 and 15 days of $\mathrm{NaCl}$ stress (Fig. 4e and f). The increase of phenolics concentration was greater under moderate stress with $150 \mathrm{mM} \mathrm{NaCl}$ compared with severe stress with $300 \mathrm{mM} \mathrm{NaCl}$ (Fig. 4a-d).

\subsection{Gene transcription under $\mathrm{NaCl}$ stress in hydroponic experiments}

PAL gene family consists of PAL1, PAL2 and PAL3, and their transcription was significantly elevated by 150 and $300 \mathrm{mM} \mathrm{NaCl}$ at day 7 and 15 (Fig. 5).

\subsection{Phenolics concentration in plants in saline plots in field trials}

Concentrations of total phenolics, flavonoid, chlorogenic acid and luteoloside in the leaves were maintained at a higher level in

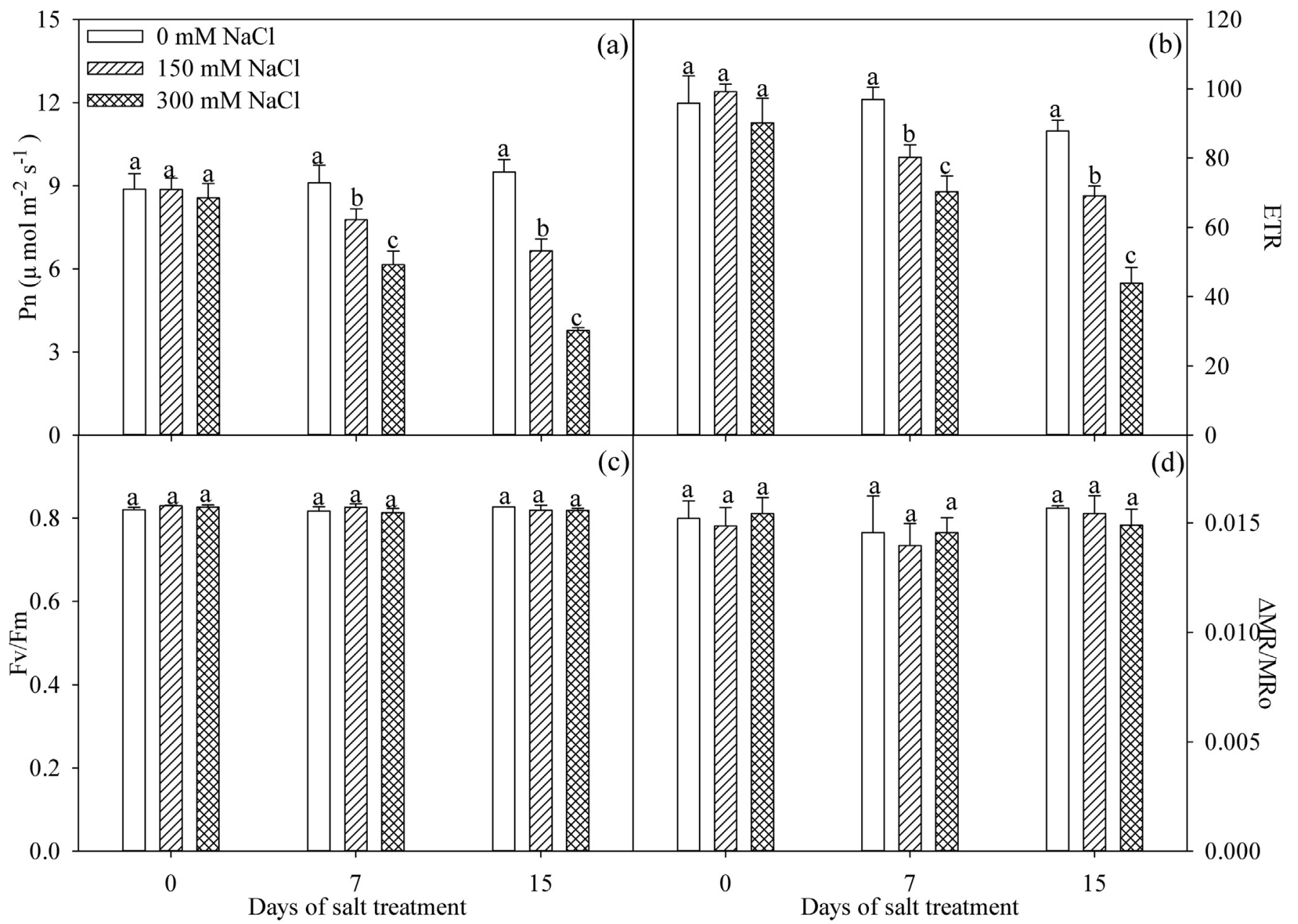

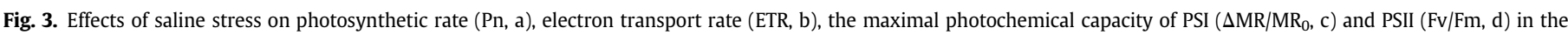
leaves of honeysuckle. Data in the figure indicate the means of four replicates $( \pm \mathrm{SD})$. Different letters on error bars indicate salt-induced significant differences at $P<0.05$. 


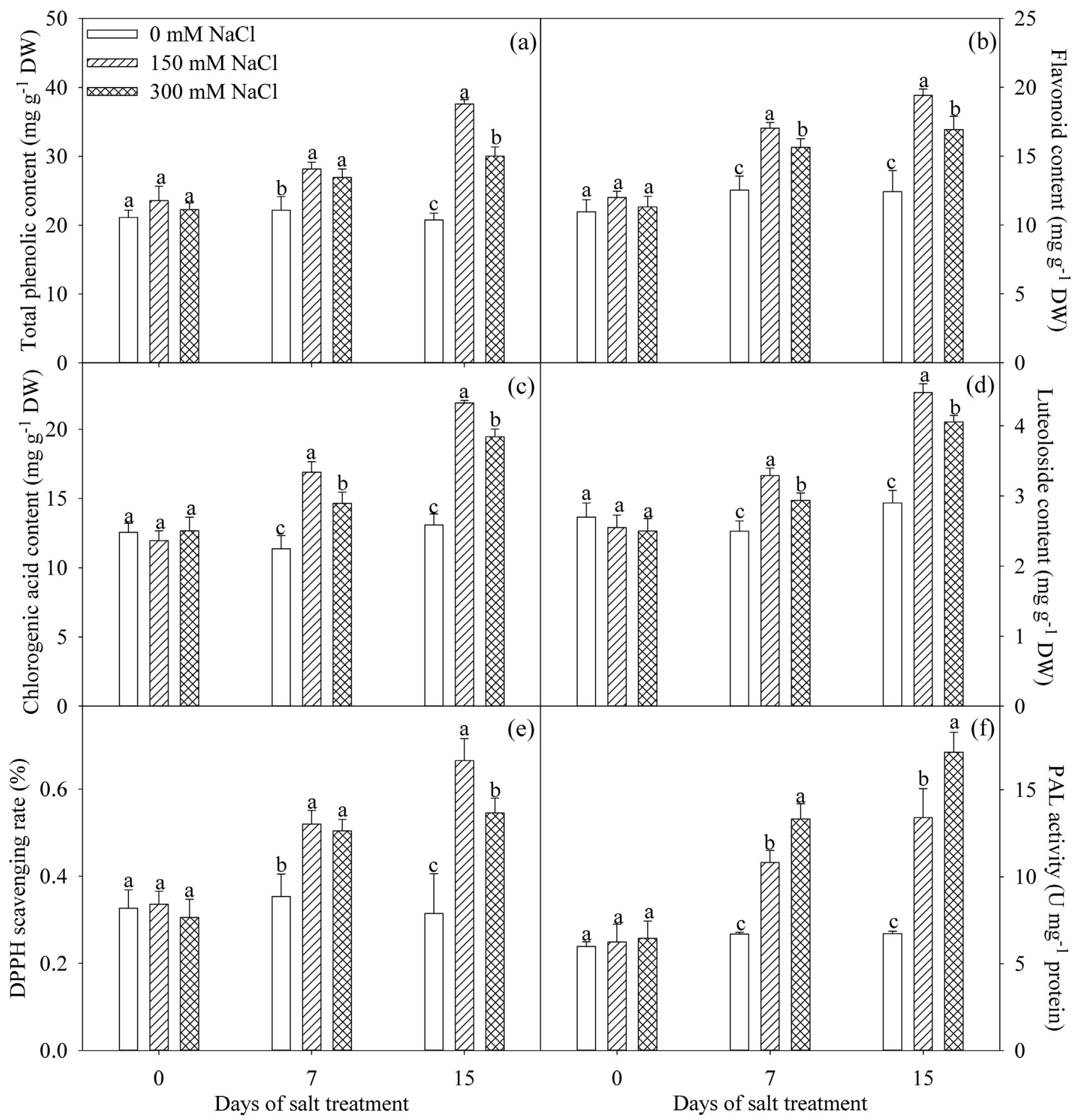

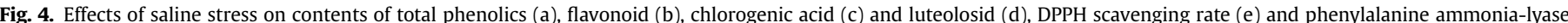

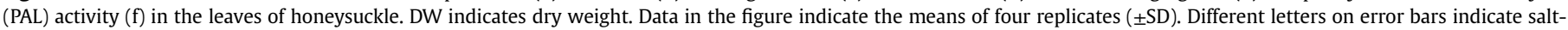
induced significant differences at $P<0.05$.

plants in saline plots compared with non-saline plots in growth seasons of 2014 and 2015 (Fig. 6a-h). The differences of their concentrations between the plants from saline and non-saline plots were insignificant in some months (Fig. 6a-h). No significant changes in MDA content were observed in the leaves of plants exposed to soil salinity (Fig. $6 \mathrm{i}$ and $\mathrm{j}$ ).

\section{Discussion}

ROS are able to bring about lipid peroxidation by initiating a chain reaction on polyunsaturated fatty acids, and MDA content representing the extent of lipid peroxidation is a classic parameter to reflect oxidative injury (Gill and Tuteja, 2010; Yan et al., 2013b). 


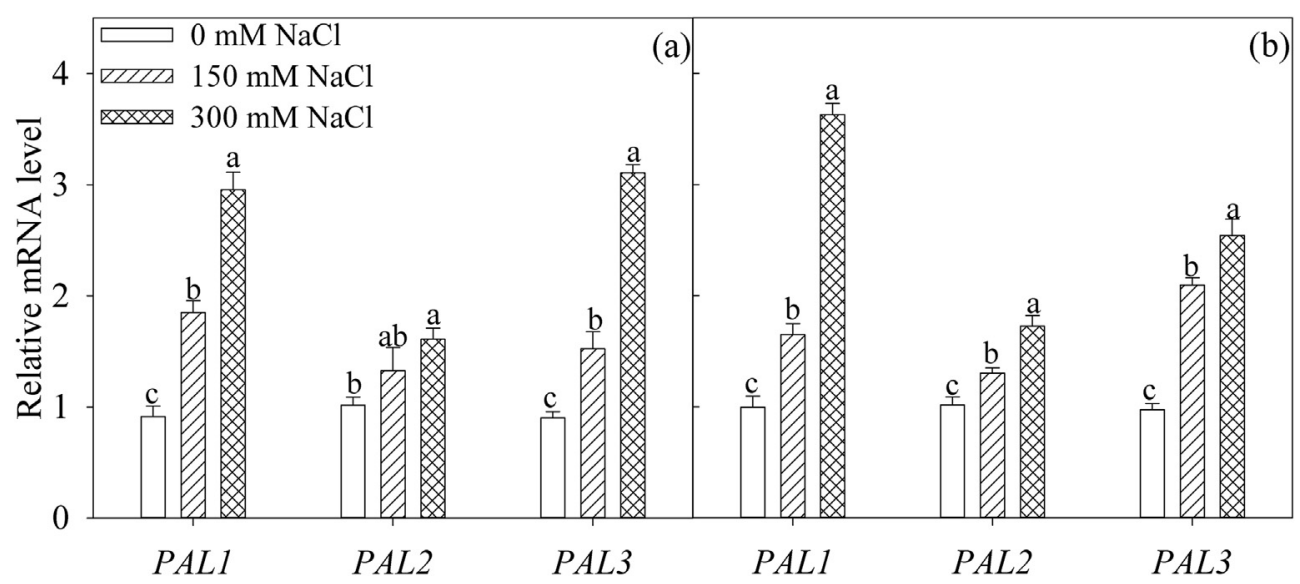

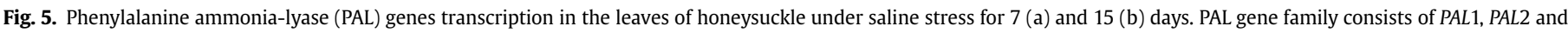
$P A L 3$. Data in the figure indicate the means of four replicates $( \pm S D)$. Different letters on error bars indicate salt-induced significant differences at $P<0.05$.

In this study, insignificant changes of MDA content suggested that oxidative injury did not occur in the leaves of honeysuckle under $\mathrm{NaCl}$ stress in the hydroponic experiments (Fig. 2f). In parallel with aggravated lipid peroxidation, oxidative stress commonly stimulates antioxidant system in plant tissues, and no induction on antioxidant system confirmed that oxidative stress was prevented without elevation of ROS production in the leaves of honeysuckle under $\mathrm{NaCl}$ stress in the hydroponic experiments (Fig. 2a-e). As widely known, photoinhibition of photosystems results from oxidization of reaction center proteins by ROS (Sonoike, 2011; Takahashi and Murata, 2008), and particularly, Oukarroum et al. (2015) demonstrated that PSI and PSII photochemical capacity was negatively correlated with ROS production. Thus, no significant changes in $\Delta \mathrm{MR} / \mathrm{MR}_{0}$ and $\mathrm{Fv} / \mathrm{Fm}$ further proved that ROS production was controlled at a normal level for preventing oxidative injury in the leaves of honeysuckle under $\mathrm{NaCl}$ stress in the hydroponic experiments (Fig. $3 \mathrm{c}$ and d). However, the inhibited $\mathrm{CO}_{2}$ assimilation under $\mathrm{NaCl}$ stress could lead to more ROS production through linear photosynthetic electron transport by inducing NADPH accumulation (Fig. 3a). Correspondingly, ROS production was suppressed by virtue of declined ETR under $\mathrm{NaCl}$ stress in the hydroponic experiments (Fig. 3b). The declined ETR was a positive response, which probably resulted from elevated dissipation of excess excitation energy by photoprotective mechanisms such as xanthophyll cycle (Brestic et al., 2015, 2016; Jahns and Holzwarth, 2012). Therefore, oxidative damage was prevented by limiting ROS production in the leaves of honeysuckle under $\mathrm{NaCl}$ stress in the hydroponic experiments.

According to traditional viewpoint, accumulation of phenolics should not appear in the leaves of honeysuckle under $\mathrm{NaCl}$ stress in light of no obvious oxidative stress. On the contrary, $\mathrm{NaCl}$ stress significantly increased phenolics concentration, and elevated genes transcription and activity of PAL in the leaves of honeysuckle in the hydroponic experiments, which was a key enzyme in the pathway of phenolic synthesis (Figs. 4 and 5). In spite of elevated DPPH scavenging rate in vitro (Fig. 4e), the accumulated phenolics rarely had the chance to scavenge ROS in vivo in absence of oxidative stress. Thus, it is surprising that much carbon was consumed to synthesize phenolics upon the decreased $\mathrm{CO}_{2}$ assimilation. A quite quantity of NADPH is required for phenolic synthesis (Grace and Logan, 2000), and Hernandez and Van Breusegem (2010) suggested that the protective role of flavonoid as energy escape valve during abiotic stresses was largely underrated in comparison to their antioxidant function. Therefore, similar to photoprotective mechanisms, phenolic synthesis helped inhibit ROS production by alleviating NADPH accumulation in the leaves of honeysuckle under $\mathrm{NaCl}$ stress. It is worth to note that increased leaf phenolics concentration without oxidative injury and antioxidant system induction under $\mathrm{NaCl}$ stress is an uncommon finding. The reason may be that $\mathrm{NaCl}$ treatments with low light in the hydroponic experiments were not beneficial to ROS production. Similarly, this uncommon finding was also reported in other medicinal plant, Swertia chirata, under mild $\mathrm{NaCl}$ stress, and the stimulation of antioxidant enzymes in this plant did not appear until salt-induced oxidative stress became greater (Abrol et al., 2012). Therefore, we suppose that antioxidant system induction stems from the increase of ROS generation, but phenolic biosynthesis is induced by NADPH accumulation.

$\mathrm{NaCl}$ stress improved leaf medicinal quality of honeysuckle by promoting accumulation of chlorogenic acid and luteolosid in the hydroponic experiments (Fig. 4c and d). Similar to other medicinal plants (Abrol et al., 2012; Colla et al., 2013; Ksouri et al., 2007; Shao et al., 2015), moderate saline stress was more beneficial to the accumulation of bioactive compounds in the leaves of honeysuckle (Fig. 4c and d), as severe stress greatly reduced carbon supply for phenolic synthesis by inhibiting $\mathrm{CO}_{2}$ assimilation. In line with $\mathrm{NaCl}$ stress in the hydroponic experiments, soil salinity also enhanced concentrations of phenolic compounds in the leaves of honeysuckle without aggravated lipid peroxidation in the field trials (Fig. 6). Thus, leaf phenolics accumulation was also an ecological mechanism in honeysuckle for the adaption to saline soil. The inconsistent result of Bautista et al. (2016) may derive from species difference and specific climatic conditions. However, we agree with them that environmental factors such as light, temperature and precipitation influence plant phenolic synthesis. Therefore, leaf phenolics concentration showed seasonal changes in the field trials (Fig. 6a-h); the insignificant difference of leaf phenolics concentration between plants in saline and non-saline plots in some months might derive from dilution effects of those environmental factors (Fig. 6a-h). Importantly, soil salinity improved leaf medicinal quality in honeysuckle by promoting accumulation of chlorogenic acid and luteolosid in the field trials (Fig. 6e-h). In combination with its phytoremediation effect on saline soil (Yan et al., 2016), honeysuckle is a promising material for planting in saline land.

In agreement with the hypothesis, leaf phenolics accumulation was improved in honeysuckle under saline stress, however, the accumulated leaf phenolics did not directly assisted antioxidant system to scavenge ROS. In addition, saline stress is beneficial to the accumulation of leaf bioactive compounds in honeysuckle. 


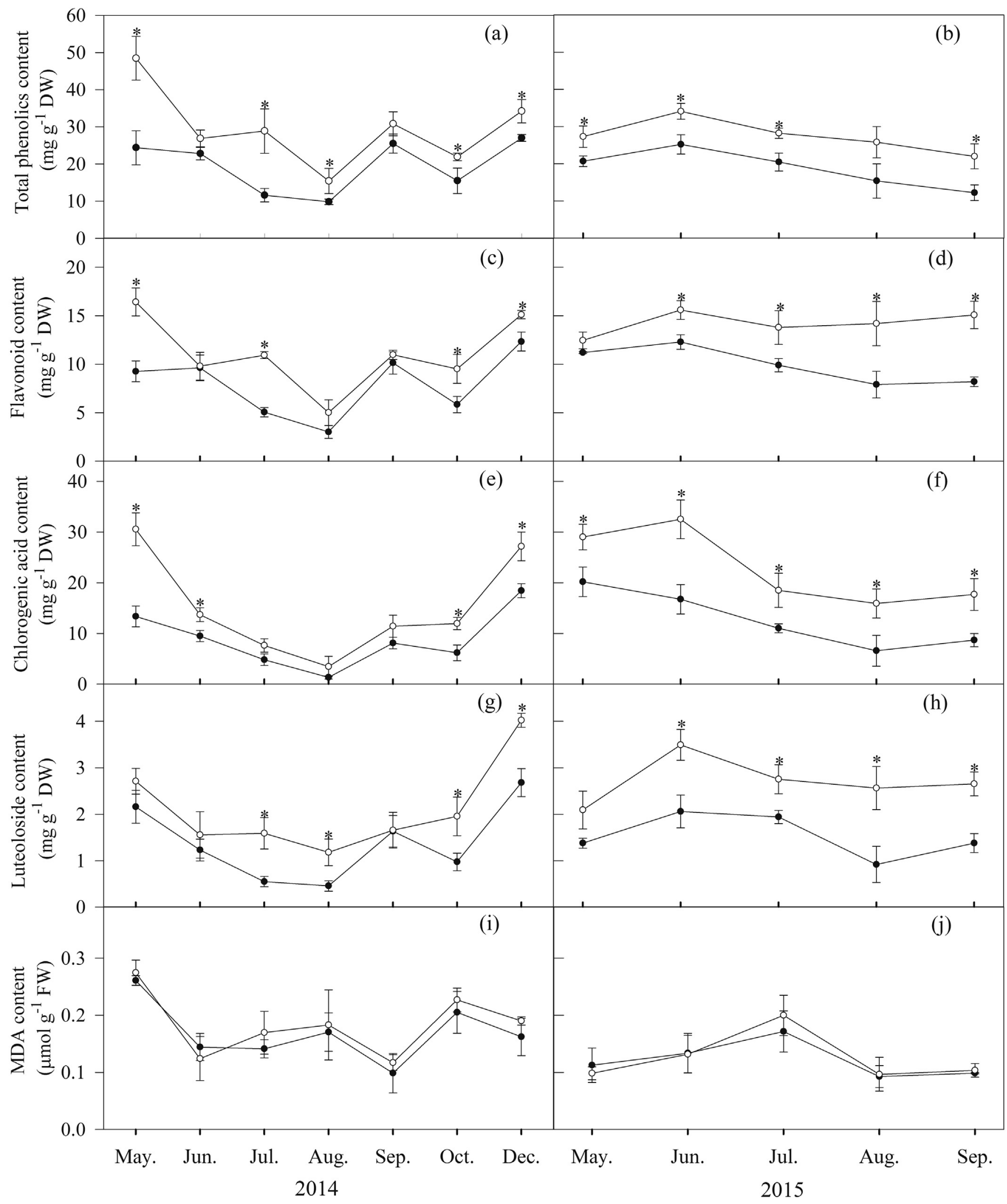

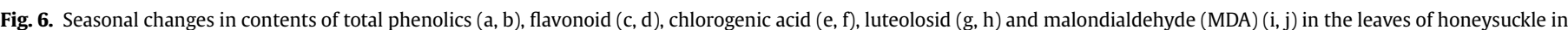

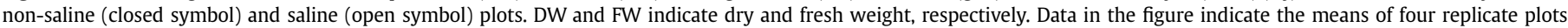
$\left( \pm\right.$ SD). Significant differences induced by salinity are indicated by asterisks: ${ }^{*} P<0.05$. 


\section{Authors contribution}

KY designed the experiments, performed data analysis, and wrote the manuscript. SZ participated in experimental design and reviewed the manuscript. LB participated in conducting the experiments. XC assisted in the construction of experimental plots.

\section{Acknowledgements}

This research was jointly supported by the National Natural Science Foundation of China (41201292), the Yantai Science and Technology Planning Project (2015ZH069) and the Opening Foundation of the State Key Lab of Crop Biology, Shandong Agricultural University (2016KF07).

\section{References}

Abrol, E., Vyas, D., Koul, S., 2012. Metabolic shift from secondary metabolite production to induction of anti-oxidative enzymes during $\mathrm{NaCl}$ stress in Swertia chirata Buch.-Ham. Acta Physiol. Plant 34, 541-546.

Asada, K., 2006. Production and scavenging of reactive oxygen species in chloroplasts and their functions. Plant Physiol. 141, 391-396.

Ashrafi, E., Razmjoo, J., Zahedi, M., Pessarakli, M., 2015. Screening alfalfa for salt tolerance based on lipid peroxidation and antioxidant enzymes. Agron. J. 107, $167-173$.

Bautista, I., Boscaiu, M., Lidon, A., Llinares, J.V., Lull, C., Donat, M.P., Mayoral, O., Vicente, O., 2016. Environmentally induced changes in antioxidant phenolic compounds levels in wild plants. Acta Physiol. Plant 38, 9.

Brestic, M., Zivcak, M., Kunderlikova, K., Sytar, O., Shao, H., Kalaji, H.M., Allakhverdiev, S.I., 2015. Low PSI content limits the photoprotection of PSI and PSII in early growth stages of chlorophyll b-deficient wheat mutant lines. Photosynth. Res. 125, 151-166.

Brestic, M., Zivcak, M., Kunderlikova, K., Allakhverdiev, S.I., 2016. High temperature specifically affects the photoprotective responses of chlorophyll b-deficient wheat mutant lines. Photosynth. Res. 130, 251-266.

Colla, G., Rouphael, Y., Cardarelli, M., Svecova, E., Rea, E., Lucini, L., 2013. Effects of saline stress on mineral composition, phenolic acids and flavonoids in leaves of artichoke and cardoon genotypes grown in floating system. J. Sci. Food Agric. 93, 1119-1127.

Falleh, H., Jalleli, I., Ksouri, R., Boulaaba, M., Guyot, S., Magne, C., Abdelly, C., 2012. Effect of salt treatment on phenolic compounds and antioxidant activity of two Mesembryanthemum edule provenances. Plant Physiol. Bioch 52,1-8.

Ferreres, F., Figueiredo, R., Bettencourt, S., Carqueijeiro, I., Oliveira, J., GilIzquierdo, A., Pereira, D.M., Valentao, P., Andrade, P.B., Duarte, P., Ros Barcelo, A., Sottomayor, M., 2011. Identification of phenolic compounds in isolated vacuoles of the medicinal plant Catharanthus roseus and their interaction with vacuolar class III peroxidase: an $\mathrm{H}_{2} \mathrm{O}_{2}$ affair? J. Exp. Bot. 62, 2841-2854.

Fini, A., Brunetti, C., Di Ferdinando, M., Ferrini, F., Tattini, M., 2011. Stress-induced flavonoid biosynthesis and the antioxidant machinery of plants. Plant Signal. Behav. 6, 709-711.

Gill, S.S., Tuteja, N., 2010. Reactive oxygen species and antioxidant machinery in abiotic stress tolerance in crop plants. Plant Physiol. Bioch 48, 909-930.

Grace, S.C., Logan, B.A., 2000. Energy dissipation and radical scavenging by the plant phenylpropanoid pathway. Proc. R. Soc. B-Biol. Sci. 355, 1499-1510.

Hernandez, I., Alegre, L., Van Breusegem, F., Munne-Bosch, S., 2009. How relevant are flavonoids as antioxidants in plants? Trends Plant. Sci. 14, 125-132.

Hernandez, I., Van Breusegem, F., 2010. Opinion on the possible role of flavonoids as energy escape valves: novel tools for nature's Swiss army knife? Plant Sci. 179, $297-301$.

Jahns, P., Holzwarth, A.R., 2012. The role of the xanthophyll cycle and of lutein in photoprotection of photosystem II. Biochim. Biophys. Acta-Bioenerg 1817,
$182-193$

Kalaji, H.M., Oukarroum, A., Alexandrov, V., Kouzmanova, M., Brestic, M., Zivcak, M., Samborska, I.A., Cetner, M.D., Allakhverdiev, S.I., Goltsev, V., 2014. Identification of nutrient deficiency in maize and tomato plants by in vivo chlorophyll a fluorescence measurements. Plant Physiol. Bioch 81, 16-25.

Karray-Bouraoui, N., Harbaoui, F., Rabhi, M., Jallali, I., Ksouri, R., Attia, H., Msilini, N., Lachaal, M., 2010. Different antioxidant responses to salt stress in two different provenances of Carthamus tinctorius L. Acta Physiol. Plant 33, 1435-1444.

Khan, M.A., Abbasi, B.H., Ahmed, N., Ali, H., 2013. Effects of light regimes on in vitro seed germination and silymarin content in Silybum marianum. Ind. Crop Prod. 46, 105-110.

Kiani-Pouya, A., 2015. Changes in activities of antioxidant enzymes and photosynthetic attributes in triticale ( $\times$ Triticosecale Wittmack) genotypes in response to long-term salt stress at two distinct growth stages. Acta Physiol. Plant 37, 72

Ksouri, R., Megdiche, W., Debez, A., Falleh, H., Grignon, C., Abdelly, C., 2007. Salinity effects on polyphenol content and antioxidant activities in leaves of the halophyte Cakile maritima. Plant Physiol. Bioch 45, 244-249.

Lister, C.E., Lancaster, J.E., Walker, J.R.L., 1996. Phenylalanine ammonia-lyase (PAL) activity and its relationship to anthocyanin and flavonoid levels in New Zealand-grown apple cultivars. J. Am. Soc. Hortic. Sci. 121, 281-285.

Maxwell, K., Johnson, G.N., 2000. Chlorophyll fluorescence - a practical guide. J. Exp. Bot. 51, 659-668.

Munns, R., Tester, M., 2008. Mechanisms of salinity tolerance. Annu. Rev. Plant Biol. 59, 651-681.

Niggeweg, R., Michael, A.J., Martin, C., 2004. Engineering plants with increased levels of the antioxidant chlorogenic acid. Nat. Biotechnol. 22, 746-754.

Oukarroum, A., Bussotti, F., Goltsev, V., Kalaji, H.M., 2015. Correlation between reactive oxygen species production and photochemistry of photosystems I and II in Lemna gibba L. plants under salt stress. Environ. Exp. Bot. 109, 80-88.

Schansker, G., Srivastava, A., Govindjee, Strasser, R.J., 2003. Characterization of the 820-nm transmission signal paralleling the chlorophyll a fluorescence rise (OJIP) in pea leaves. Funct. Plant Biol. 30, 785-796.

Shao, Y., Gao, J., Wu, X., Li, Q., Wang, J., Ding, P., Lai, X., 2015. Effect of salt treatment on growth, isoenzymes and metabolites of Andrographis paniculata (Burm. f.) Nees. Acta Physiol. Plant 37, 35.

Sonoike, K., 2011. Photoinhibition of photosystem I. Physiol. Plant 142, 56-64.

Takahashi, S., Murata, N., 2008. How do environmental stresses accelerate photoinhibition? Trends Plant Sci. 13, 178-182.

Yan, K., Chen, P., Shao, H.B., Zhao, S.J., 2013a. Characterization of photosynthetic electron transport chain in bioenergy crop Jerusalem artichoke (Helianthus tuberosus L.) under heat stress for sustainable cultivation. Ind. Crop Prod. 50, 809-815.

Yan, K., Chen, W., He, X.Y., Zhang, G.Y., Xu, S., Wang, L.L., 2010. Responses of photosynthesis, lipid peroxidation and antioxidant system in leaves of Quercus mongolica to elevated $\mathrm{O}_{3}$. Environ. Exp. Bot. 69, 198-204.

Yan, K., Shao, H.B., Shao, C.Y., Chen, P., Zhao, S.J., Brestic, M., Chen, X.B., 2013b. Physiological adaptive mechanisms of plants grown in saline soil and implications for sustainable saline agriculture in coastal zone. Acta Physiol. Plant 35, 2867-2878.

Yan, K., Wu, C., Zhang, L., Chen, X., 2015. Contrasting photosynthesis and photoinhibition in tetraploid and its autodiploid honeysuckle (Lonicera japonica Thunb.) under salt stress. Front. Plant Sci. 6, 227.

Yan, K., Xu, H., Zhao, S., Shan, J., Chen, X., 2016. Saline soil desalination by honeysuckle (Lonicera japonica Thunb.) depends on salt resistance mechanism. Ecol Eng. 88, 226-231.

Zhang, L., Ma, H., Chen, T., Pen, J., Yu, S., Zhao, X., 2014. Morphological and physiological responses of cotton (Gossypium hirsutum L.) plants to salinity. PLoS One 9, e112807.

Zhang, Q.L., Li, J., Wang, C., Sun, W., Zhang, Z.T., Cheng, W.M., 2007. A gradient HPLC method for the quality control of chlorogenic acid, linarin and luteolin in Flos Chrysanthemi Indici suppository. J. Pharm. Biomed. 43, 753-757.

Zhao, G., Li, S., Sun, X., Wang, Y., Chang, Z., 2015. The role of silicon in physiology of the medicinal plant (Lonicera japonica L.) under salt stress. Sci. Rep. 5, 12696.

Zhu, J.K., 2016. Abiotic stress signaling and responses in plants. Cell 167, 313-324.

Zhou, C.H., Sun, C.D., Li, X., 2007. Study on method for flavonoids determining of plant rich in chorogenic acid. Plant Physiol. Commun 43, 902-904 (In Chinese). 